\title{
On $k$-Transitive Closures of Directed Paths
}

\author{
Krzysztof Pszczoła \\ Institute of Mathematics, Jan Kochanowski University, Kielce, Poland \\ Email: krzysztof.pszczola@ujk.edu.pl
}

Received 31 December 2014; accepted 16 October 2015; published 19 October 2015

Copyright (C) 2015 by author and Scientific Research Publishing Inc.

This work is licensed under the Creative Commons Attribution International License (CC BY).

http://creativecommons.org/licenses/by/4.0/

(c) (i) 0 pen Access

\section{Abstract \\ In this paper we study the structure of $k$-transitive closures of directed paths and formulate several properties. Concept of $\boldsymbol{k}$-transitive orientation generalizes the traditional concept of transitive orientation of a graph.}

\section{Keywords}

\section{Digraph, Transitive Graph, $k$-Transitive Digraph, $k$-Transitive Closure of an Oriented Graph}

\section{Introduction}

We use the standard notation. By an edge we mean an unoriented pair of vertices, and by an arc we mean an oriented pair of vertices. For a given graph $G, V(G)$ and $E(G)$ denote the set of its vertices and the set of its edges, respectivly. For a digraph $G$, we write $A(G)$ for the set of its arcs. By an oriented graph we mean such a digraph that if $(a, b)$ is an arc, then $(b, a)$ is not. All graphs and digraphs in this paper are finite.

\section{Motivation}

Orientation of a graph $G$ is called transitive if for every $(a, b) \in A(G),(b, c) \in A(G)$, also $(a, c) \in A(G)$. This concept was studied by many authors in numerous papers, see the survey [1] for example. The concept of transitive orientation was generalized in several ways in [2] and [3], [4] and [5], and other papers.

A digraph is called $k$-transitive if every directed path of the length $k$ has a shortcut joining the beginning and the end of this path. In other words, if $\left(v_{0}, \cdots, v_{k}\right)$ is a path in the digraph $G$, then $\left(v_{0}, v_{k}\right) \in A(G)$.

Note that our term " $k$-transitive" coresponds to " $(k, 1)$-transitive" in [2] and [3].

A $k$-transitive closure of an oriented graph $G=(V, A)$ is an oriented graph $\mathrm{T}_{k} \mathrm{C}(G)$ such that

(1) $V\left(\mathrm{~T}_{k} \mathrm{C}(G)\right)=V(G)$

(2) $A(G) \subset A\left(\mathrm{~T}_{k} \mathrm{C}(G)\right)$ 
(3) $\mathrm{T}_{k} \mathrm{C}(G)$ is $k$-transitive.

(4) it has the minimal (by inclusion) set of arcs among all graphs with the above stated properties.

Observe that there are oriented graphs for which the $k$-transitive closure does not exist. For example in a cyclically oriented cycle $C_{k+1}$ it is not possible to add arcs to fulfill the condition (3).

If the $k$-transitive closure does exist for some oriented graph, it is unique.

Note that this definition is a partial answer to the point (4) in ([2], p. 41).

The aim of this paper is to describe $k$-transitive closures of directed paths.

\section{Structure of the $k$-Transitive Closure of the Directed Path}

Instead of $\mathrm{T}_{k} \mathrm{C}\left(P_{n-1}\right)$ we write $(k: n)$ to denote the $k$-transitive closure of an oriented path on $n$ vertices. We label the vertices by natural numbers $1,2, \cdots, n$ and assume that $(i, i+1) \in A\left(P_{n-1}\right)$ for $1 \leq i<n$.

Although the graph $(k: n)$ is oriented, some of the properties will be stated for simple graphs obtained by "forgetting" the orientation. We belive that it is clear from the context, but to be precise, for the unoriented case we write $[k: n]$.

In this paper by a degree sequence of a graph $[k: n]$ we mean a sequence $(\operatorname{deg}(1), \cdots, \operatorname{deg}(n))$. (In/out) degree sequence of a graph $(k: n)$ is defined in a similiar way.

Observe that $[2: n]$ is just the complete graph $K_{n}$, and $(2: n)$ is the tournament on $n$ vertices.

The starting point in a construction of the $k$-transitive closure of the path $P_{n-1}$ is to add arcs $(i, i+k)$. Then we add arcs $(i, i+2 k-1)$, at the next stage arcs $(i, i+3 k-2)$, and so on. This construction shows that for every $k, n \in \mathbb{N},(k: n)$ is well defined.

The key observations are:

3.1 Fact. Adding one vertex to the path adds only arcs ending in this new vertex. In other words, $(k: n)$ is an induced subgraph of $(k: n+1)$.

3.2 Fact. In the graph $(k: n),(i, j) \in A((k: n))$ iff $j-i=1+l(k-1)$ for some $0 \leq l \leq(n-2) /(k-1)$.

Proof. It follows directly from the construction described above that

$$
\{(i, j): \exists 0 \leq l \leq(n-2) /(k-1), j-i=1+l(k-1)\} \subset A((k: n)) .
$$

To show the other inclusion we use the induction on $n$. First observe that for $n \leq k$ all arcs are of the form $(i, i+1)$. Assume that all arcs in $(k: n-1)$ have the length $1+l(k-1)$ for some $l \geq 0$. To obtain a $k$-shortcut in $(k: n)$ we need $k$ arcs, each of them of length $1+l_{i}(k-1), l_{i} \geq 0$. So

$$
j-i=1+l_{1}(k-1)+1+l_{2}(k-1)+\cdots+1+l_{k}(k-1)=k+(k-1)\left(l_{1}+l_{2}+\cdots+l_{k}\right)=1+(k-1)\left(1+l_{1}+l_{2}+\cdots+l_{k}\right) \text {. }
$$

In Figure 1 we present the graph $(5: 11)$ as an example.

\section{Some Properties}

From the observations mentioned above, we conclude several properties of graphs $(k: n)$ and $[k: n]$.

4.1 Fact. For $n \leq k, \mathrm{~T}_{k} \mathrm{C}\left(P_{n-1}\right)=P_{n-1}$. So for $n \leq k$, the graph $(k: n)$ is just the path $P_{n-1}$. We can observe the following block structure in indegree/outdegree sequences of graphs $(k: n)$ :

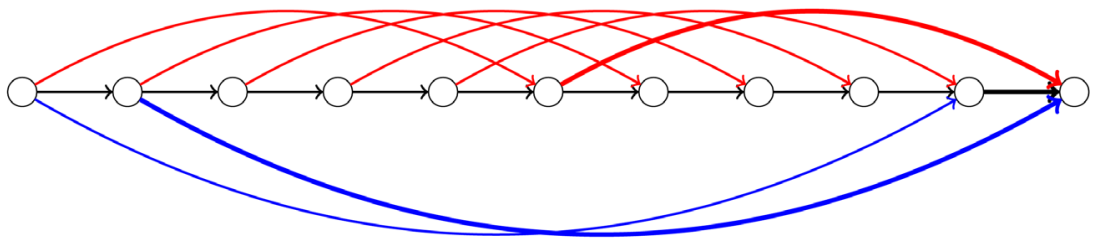

Figure 1. The graph $(5: 11)$. All arcs ending in the last vertex are drawn with thick lines. 
4.2 Theorem. Let $n=2+l(k-1)+m$ for some $l \in \mathbb{N}$ and $0 \leq m<k-1$. In the oriented graph $(k: n)$ the indegree sequence is built from uniform "blocks" of length $k-1$ and has the form

$$
(0, \underbrace{1, \cdots, 1}_{k-1 \text { times }}, \underbrace{2, \cdots, 2}_{k-1 \text { times }}, \cdots, \underbrace{l, \cdots, l}_{k-1 \text { times }}, \underbrace{l+1, \cdots, l+1}_{m+1 \text { times }}) .
$$

Similarly, the outdegree sequence is built from uniform "blocks" of length $k-1$ and has the form

$$
(\underbrace{l+1, \cdots, l+1}_{m+1 \text { times }}, \underbrace{l, \cdots, l}_{k-1 \text { times }}, \cdots, \underbrace{2, \cdots, 2}_{k-1 \text { times }}, \underbrace{1, \cdots, 1}_{k-1 \text { times }}, 0) .
$$

Proof. The proof follows from Facts 3.2 and 3.1. We prove the part concerning the indegree sequence. First note that the indegree of the first vertex is 0 . For the next $k-1$ vertices there is no arcs ending in them other than the arcs in the initial path, so their indegree is 1 . First vertex of indegree 2 is the $(k+1)$-th vertex. First vertex of indegree 3 is the $2 k$-th vertex, and first vertex of indegree $j$ is the $((j-1)(k-1)+2)$-th vertex.

The proof for the outdegree sequence is similiar; we just start from the last vertex.

4.3 Corollary. The graph $[k: 2+l(k-1)]$ is $(l+1)$-regular for every $l \in \mathbb{N}$.

Proof. This is a consequence of Theorem 4.2; just observe that summing up the indegree and outdegree sequences gives the constant sequence $(l+1, \cdots, l+1)$.

4.4 Corollary. For every $l \in \mathbb{N}$, and for every $0<m<k-1$, all vertices of the graph $[k: 2+l(k-1)+m]$ has degree $l+1$ or $l+2$. Morover, if we put $a=l+1$ and $b=l+2$, the degree sequence is built from "blocks" of the form

$$
(a, \underbrace{b, \cdots, b}_{m \text { times }}, \underbrace{a, \cdots, a}_{k-m-2 \text { times }})
$$

repeated to get the sequence of the length $2+l(k-1)+m$. Note that the last "block" has the length $m+2(\bmod (k-1))$.

Proof. This is another consequence of Theorem 4.2.

4.5 Corollary. For every $l \in \mathbb{N}$, and for every $0 \leq m<k-1$, in the graph $[k: 2+l(k-1)+m]$ there are $m(l+1)$ vertices of degree $l+2$ and $l(k-m-1)+2$ vertices of degree $l+1$.

As an example, below are the degree sequences for 5-transitive closures of the paths on 10, 11, 12, 13 and 14 vertices:

- for $[5: 10]:(3,3,3,3,3,3,3,3,3,3) ; 10=2+2(5-1)+0, l=2$ and $m=0$, by Corollary 4.3 this graph is $2+1=3$ regular;

- for $[5: 11]:(3,4,3,3,3,4,3,3,3,4,3) ; 11=2+2(5-1)+1, \quad l=2$ and $m=1$, by Corollary 4.4 this sequence is built from repeated blocks $(3,4,3,3)$;

- for [5:12]: $(3,4,4,3,3,4,4,3,3,4,4,3) ; 12=2+2(5-1)+2, \quad l=2$ and $m=2$, by Corollary 4.4 this sequence is built from repeated blocks $(3,4,4,3)$;

- for $[5: 13]:(3,4,4,4,3,4,4,4,3,4,4,4,3) ; 13=2+2(5-1)+3, l=2$ and $m=3$, by Corollary 4.4 this sequence is built from repeated blocks $(3,4,4,4)$;

- for $[5: 14]:(4,4,4,4,4,4,4,4,4,4,4,4,4,4) ; 14=2+3(5-1)+0, l=3$ and $m=0$, by Corollary 4.3 this graph is $3+1=4$ regular.

Recall that by degree of a vertex $v$ in a digraph we mean a pair (indegree $(v)$, outdegree $(v)$ ).

For oriented graphs $(k: n)$ we can observe the following: 
4.6 Corollary. Every constant subsequence in the degree sequence of the non regular graph $[k: n]$ is also the constant subsequence in the degree sequence of the oriented graph $(k: n)$.

For example, the degree sequence for $[5: 12]$ is $(3,4,4,3,3,4,4,3,3,4,4,3)$, and the degree sequence for $(5: 12)$ is $((0,3),(1,3),(1,3),(1,2),(1,2),(2,2),(2,2),(2,1),(2,1),(3,1),(3,1),(3,0))$.

Recall that an oriented graph $G$ is irregular if for every two vertices $v_{i}, v_{j} \in V(G), v_{i} \neq v_{j}$, their degrees are different.

Straightforward consequence of Corollary 4.6 is that graphs $(k: n)$ for $k>3$ are not irregular. The natural question is: are the graphs $(3: n)$ irregular? The answer is:

4.7 Theorem. Oriented graphs $(3: n)$ are irregular iff $n$ is odd.

Proof. By Theorem 4.2, pairs of vertices $2 i, 2 i+1$ for $1 \leq i<n / 2$ have the same indegree. Because for the even $n$ the graph $[3: n]$ is regular, so $(3: n)$ is not irregular if $n$ is even.

Also by Theorem 4.2, indegree $(2 i)=$ indegree $(2 i-1)+1$ for $1 \leq i<n / 2$. By Corollary 4.4 , for the odd $n$ the degree sequence of the graph $[3: n]$ is of the form $(a, b, a, \cdots, b, a)$. So for $n$ odd, if for some two vertices their total degrees are equal then its indegrees are different. Hence $(3: n)$ is irregular if $n$ is odd.

Recall that the tournament $(2: n)$ is irregular for any $n$.

\section{Density}

By density of the graph $G,|V(G)|=n$, we mean ratio "number of edges in the graph $G$ " / "number of edges in complete graph $K_{n}$ ”; in symbols $\operatorname{Dens}(G)=E(G) / E\left(K_{n}\right)=2 E(G) /(n(n-1))$.

Recall then for every even $n$, a graph $[3: n]$ is $\frac{n}{2}$-regular. We have $\frac{1}{4} n^{2}$ edges. So for even $n$, $\operatorname{Dens}([3: n])=\frac{1}{2} \frac{n}{n-1}$.

For every odd $n$, in a graph $[3: n]$ there are $\left\lceil\frac{n}{2}\right\rceil$ vertices of degree $\left\lfloor\frac{n}{2}\right\rfloor$ and $\left\lfloor\frac{n}{2}\right\rfloor$ vertices of degree $\left\lceil\frac{n}{2}\right\rceil$. We have $\frac{1}{4}\left(n^{2}-1\right)$ edges. So for odd $n$, Dens $([3: n])=\frac{1}{2} \frac{n+1}{n}$.

Observe that in both cases the density is bigger then $1 / 2$ and

$$
\lim _{n \rightarrow \infty} \operatorname{Dens}([3: n])=1 / 2 \text {. }
$$

We have the following:

5.1 Theorem. For $k>2$,

$$
\lim _{n \rightarrow \infty} \operatorname{Dens}([k: n])=1 /(k-1) .
$$

Proof. By Corollary 4.5,

$$
\operatorname{Dens}([k:(2+l(k-1)+m)])=\frac{m(l+1)(l+2)+(2+l(k-m-1))(l+1)}{n(n-1)} .
$$

By standard calculation we get

$$
\begin{aligned}
\operatorname{Dens}([k:(2+l(k-1)+m)]) & =\frac{(l+1)(m(l+2)+(2+l(k-m-1)))}{(2+l(k-1)+m)(1+l(k-1)+m)} \\
& =\frac{l^{2}(k-1)+l(k+2 m+1)+2(m+1)}{l^{2}(k-1)^{2}+l(k-1)(2 m+3)+(m+1)(m+2)} .
\end{aligned}
$$


Recall that $n=2+l(k-1)+m$, where $k$ and $m$ are fixed, so when $l \rightarrow \infty$, then also $n \rightarrow \infty$. So

$\lim _{n \rightarrow \infty} \operatorname{Dens}([k: n])=1 /(k-1)$.

Obviously, for $k=2$, Dens $([2: n])=1$.

\section{Open Problems}

The main open problem concerning $k$-transitive closures in general, is to state what properties of an oriented graph $G$ guarantee the existence of $\mathrm{T}_{k} \mathrm{C}(G)$.

There are also some other special classes of oriented graphs, such as cycles (with different orientations) and trees, for which there is a chance to obtain interested properties for their $k$-transitive closures.

\section{Acknowledgements}

We acknowledge the support by the UJK grant No. 612439.

Some of the results contained in this paper were presented at the 5th Polish Combinatorial Conference, Będlewo, September 22-26, 2014. The author wants to express his thanks to Professor Zsolt Tuza for pointing to valuable references.

\section{References}

[1] Kelly, D. (1985) Comparability Graphs. In: Rival, I., Ed., Graphs and Order. The Role of Graphs in the Theory of Ordered Sets and Its Applications, North Holland, Dordrecht, 3-40. http://dx.doi.org/10.1007/978-94-009-5315-4_1

[2] Gyárfás, A., Jacobson, M.S. and Kinch, L.F. (1988) On a Generalization of Transitivity for Digraphs. Discrete Mathematics, 69, 35-41. http://dx.doi.org/10.1016/0012-365X(88)90175-6

[3] Tuza, Z. (1994) Characterization of $(m, 1)$-Transitive and (3,2)-Transitive Semi-Complete Directed Graphs. Discrete Mathematics, 135, 335-347. http://dx.doi.org/10.1016/0012-365X(94)00060-V

[4] Hernández-Cruz, C. (2012) 3-Transitive Digraphs. Discussiones Mathematicae Graph Theory, 32, 205-219. http://dx.doi.org/10.7151/dmgt.1613

[5] Hernández-Cruz, C. and Montellano-Ballesteros, J.J. (2014) Some Remarks on the Structure of Strong $k$-Transitive Digraphs. Discussiones Mathematicae Graph Theory, 34, 651-671. http://dx.doi.org/10.7151/dmgt.1765 